\title{
A KNOWLEDGE-BASED METHOD FOR THE VALIDATION OF MILITARY SIMULATION
}

\author{
Feiyan Min \\ Ping Ma \\ Ming Yang \\ Control and Simulation Center \\ Harbin Institute of Technology \\ Harbin, Heilongjiang 150001, P.R. CHINA
}

\begin{abstract}
The validation of modern military simulation relies heavily on the opinions of military experts, and it makes the validation task exhaustive and time-consuming. The knowledgebased methods can be applied for these problems. There are three kinds of knowledge sets in military simulation validation, namely, domain knowledge, inference knowledge and validation task knowledge. By analyzing the context of these knowledge, three types of knowledge models are developed. Based on these knowledge models, the implement of knowledge-based system is detailed. However, this validation system can be practical for the validation of military simulation by enriching the knowledge base.
\end{abstract}

\section{INTRODUCTION}

The DMSO Recommended Practice Guide (2001) defines validation of simulations as "the process of determining the degree to which a model or simulation is an accurate representation of the real world from the perspective of the intended uses of the model or simulation." For decades, a variety of validation techniques have been developed and employed in simulation model validation (Balci 1989, 1998; Kleignen 1999; Sargent 2004; Law and Kelton 2000). But these techniques, both objective and subjective types, cannot always be applied in military simulation exercise conveniently.

The challenges for the validation of military simulation falls into several categories, such as mastering the complexity in both structures and behaviors of military system, collection validation data and information among plenty of application domains and struggling with limited validation time and funds. Furthermore, in modern warfare simulation, there is not usually enough real reference date about military system. The main approaches to determine validity of simulation exercise are based on the experience of experts. However, these experience is often incomplete, informal biased, and hard to utilized. These experts experience based methods often take the way of expert review by carefully planning and organizing. And they can be very exhaustive and time-consuming as the simulation scenario become gigantic and complex.

One feasible approach for these problems is the knowledge-based validation system. In fact, several expert systems for the validation of simulations among different application domains have been proposed during the last twenty years. For example, Findler, and Mazur (1990) have designed a system for the automatic verification and validation of discrete event simulation models. By defining the features of most typical mistakes involved in modeling and simulation processes, this system can easily pick out any possible mistakes. Birta and Ozmizrak (1996) have proposed a rule-based system suitable for both continuous and discrete event simulation. They abstract simulation validation as a comparing and decision process, and conclude some validity rules for each kind of validation task. Apparently, this methods cannot be adapted for warfare cases, for the complex knowledge forms and validation processes involved in military exercise validation. In addition, Hopkinson and Sepulveda (1995) also propose an expert system for the evaluation of trainee's performance. Their expert system was developed using case-based reasoning to evaluate the trainee's decisions as the simulation progressed by identifying invalid techniques. In conclusion, all these only pay attention to the structural and formal kinds of knowledge, but neglect the complex, informal and obscure information that are of gigantic size, and may be more helpful. Another defect of these methods is that they pay little consideration to the complicated validation processes, and regard them only as a comparing and decision process. These shortcomings make these expert systems hard to be applied in military simulation domains.

In this paper, we will introduce a knowledge-based validation method suitable for military simulation exercise. All the knowledge for the validity decision among different domains are collected proper form. These knowledge, namely, domain knowledge, inference knowledge and vali- 


\section{Min, Ma, and Yang}

dation task knowledge are abstracted as different kinds of knowledge models. Base on these knowledge models, a prototype system is developed. As more and more domain knowledge is collected and inserted in this system, a practical system can be produced. In addition, all the classical validation methods, both objective and subjective can be employed in this system. An important advantage of this system is that it automate the validation process, so the validation can be taken very quickly. In this paper, we will focus on the construction of knowledge models for military simulation exercise, and the implement of prototype system. The introduction about the complex characterization of military simulation knowledge context and their acquisition can be found in (Min, Ma, and Yang 2007).

The structure of this paper is as follows. In section 2, we will detail the emerging challenges military simulation exercise validation. And in section 3, an overview of our knowledge-based method is given, together with some key techniques. Section 4 discusses the constructions of three kinds of knowledge models with an example. Finally, the implement of prototype system is introduced, and also discusses some key problems with the development of practical application systems.

\section{CHALLENGE FOR MILITARY SIMULATION VALIDATION}

For more than ten years, we have dedicated our research to the application of these techniques in military simulation validation (Fang, Yang, and Wang 2005; Song, Yang, and Ma 2005; Zhang, Yang, and Li 2002). However, these techniques, mainly among subjective and objective types, were not always as powerful as they should be, especially when the military systems become comparatively complex and not enough data are available.

The objective methods mostly rely on statistical techniques, and these approaches typically require that the system are observable. The data of particular variables are collected as the simulation model is running, these data are then statistically analyzed and compared with those data from real system.

For military simulation, this statistical approach can be considerably useful for the validation of explicit and quantitative variables, such as weapon's rate of fire, the hit/miss ratio, the speed of a vehicle over a particular terrain, etc. Statistical techniques can be insightful for the analysis of weapon and combat level models, but not so powerful for mission or battle level models. In higher level military simulation systems, it cannot always quantitate the variables and behavior of interest for statistical analyze. For example, in the case of sitzkrieg, no apparent effect appears as plenty of troops and weapons are put in the battle in both of the battling parts, and this process will last for some unexpect time, for the validation of such simulation system, it was hard to extract rational quantitative variables for statis- tical analyze. In addition, and there are also observable issues regard the variables of real system (Fang, Yang, and Wang 2005), the data from real system can not be complete, and there are variety of conditions under which military model is running, but real system cannot always match. Furthermore, when a simulation includes models of future or imagine models, the expect output cannot be specified and statistical analyze become impossible.

On the other hand, the subjective methods rely heavily on expert opinions (Sargent 2004). As the simulation model is running, the experts analysis the behavior and result, compare it with their experience, and make evaluation decision. Apparently, this subjective methods can be employed for validation tasks in which quantitative data of real system are not available, especially for the validation of future or imagine models, which is common in military simulation.

However, there are also several disadvantages with subjective validation methods. First, some aspects of the system may be inadvertently overlooked or biased. Military simulation systems usually contain hundreds of entities, including plenty of different kinds of weapons and troops, and the terrain and weather conditions are considered. In the meantime, performances of different type of weapons will take very different features, and behavior of each entity varies with their configurations and running conditions. In such case, validation based on domain experts experience cannot be done totally unbiased. The second disadvantage lies on the dependence of experts. Different expert may make different decisions, then problems come up with which experts to ask and how many experts will be include for particular tasks. A third disadvantage of this methods is time and resource consuming, and cannot be utilized for real-time validation. In subjective validation, every tailored evaluation task will be considered one after another, as the military system scales up, the number of evaluation tasks will grow exponentially, and months of time is usually required. Despite of these drawbacks, the use of expert experience provide a valuable methods for the validation of aspects simulation model which there is no alternative methods, especially for the validation of military simulation system.

Another problem with military simulation validation is around the organizing and management of validation processes. There are usually several steps in military simulation exercise evaluation, and each step involves certain number of tasks. As the exercise system become more complex, the organizing and management of validation usually become considerable hard, and even impossible.

\section{KNOWLEDGE-BASED METHOD FOR THE VALIDAITON OF MILITARY SIMULATION}

To struggle with the embarrassment emerging in military simulation validation, we attempt to utilize the knowledgebased way. Our method, is essentially to automate the both 


\section{Min, Ma, and Yang}

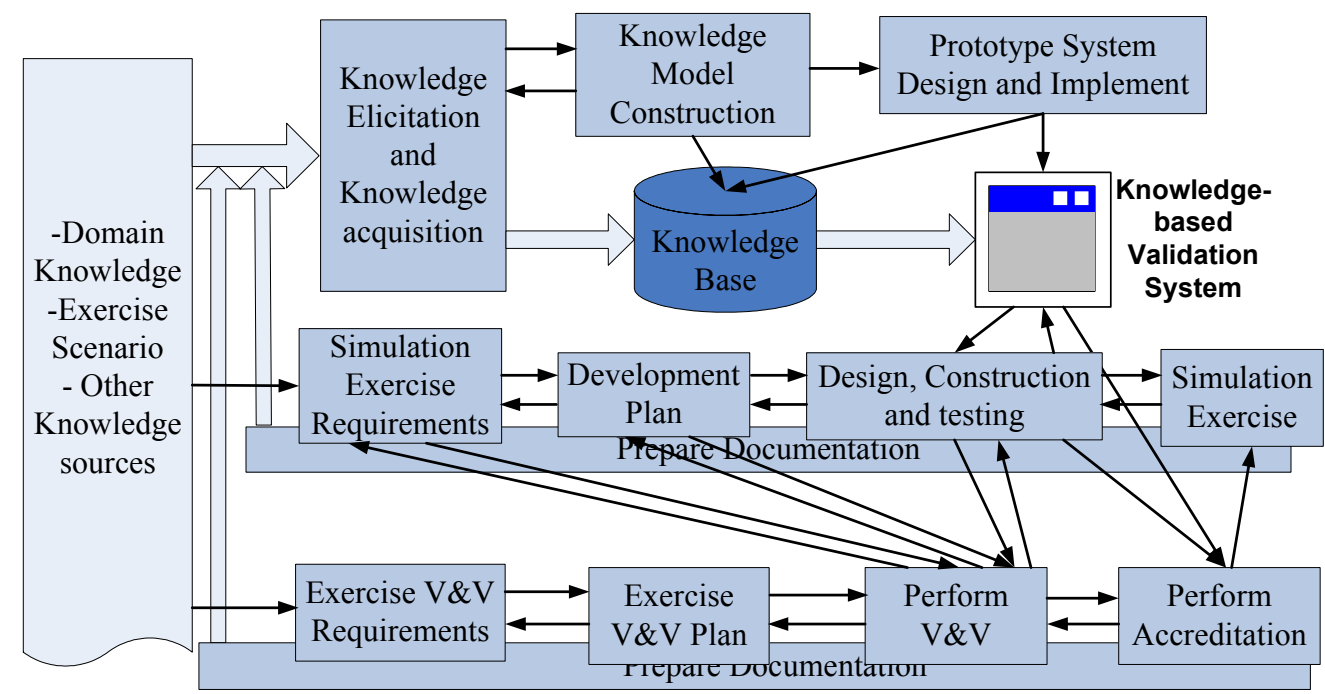

Figure 1: This figure shows the knowledge transformation process together with simulation exercise and evaluation. The wide arrowhead shows knowledge flow and transformation.

the objective and subjective techniques in a uniform framework, and all the information that could be used for validity decision are to be extracted and employed in the systems. These information, termed as domain knowledge, among from observed date to any kinds of expert experience, present tremendous opportunities and challenges for the validation of military simulation. One advantage of this method is that it attempts to formally collect all available information about any aspects of the expected behaviors and then compare the this information with the testing data. Another advantage is that this approach can be easily automated so that test results can very quickly be analyzed, which make real-time evaluation of military system feasible.

The idea to utilize knowledge for the validation of simulation model have been proposed for more than twenty years, and several expert systems have been applied in some particular areas, but few can be applied to the evaluation task of military simulation directly. One main shortcoming of these methods is that they only pay attention to the structural and formal kinds of knowledge, but neglect the complex, informal and obscure information that are of gigantic size, and may be more helpful. Another defect of these methods is that they pay little consideration to the complicated validation processes, and regard them only as a comparing and decision process. For example, Birta and Ozmizrak's system is a typical rule-based system, the author pay lots of attention to the technical practicability of the rule-based system, but not the validation knowledge and operational processes. For the military simulation, the observed data and expert experience can be comparatively complex, and the analysis and validation process usually involves several informal steps. This rule-base system cannot be applied in military simulation validation. On the other hand, Hopkinson and Sepulveda's system is in essential a case-based system, it was constrained by the number of available cases. In the mean time, the authors did not discuss the testing plan problem, either.

In our opinion, the validation of military simulation should be regarded as a knowledge-intensive process. The information involved and their transformation processes should all be paid consideration. This knowledge transformation process together with simulation exercise and evaluation are displayed in Figure 1. Our long-term research objective is to automate the whole evaluation process with knowledge-based method. However, our discussion here will focus on the validation topic.

In knowledge-based simulation validation, domain knowledge detail the characterizations of validity models, i.e., they are the reference information to make validity decision. Generally, there may be several different resources, such as observed data from real systems, every kind of formulations, and expert experience, etc.. For military simulation validation, domain knowledge can be very complex and heavily depend on exercise objective. In fact, this part of knowledge, has got the most considerations in validation system design. For the complex characterization of military domain knowledge, its elicitation and acquisition is very difficult and time consuming. We advise to carry this knowledge acquisition in three steps, namely, context definition, description and discussion. Within each step, some guidelines and proper techniques are proposed. To master the complexity of military system, the decomposition of system is necessary. The issue of complex system and structure decomposition have been discussed for a long time, especially for the decomposition of military system (Zeigler 1987; Courtois 1985; Hofmann 2004). Most aspects of military system can be decomposed into several more simple and explicit component with regard of some guidelines. For the purpose of validation, we usually decompose system according to their structure and behavior, 
namely, structural and behavior decomposition. As result of decomposition, there are usually several aspects and components to be validate, we term each of this a validation task. These validation tasks, mainly fall into two different types, structure validation and behavior validation (Barlas 1996; Barlas and Kanar 1999; Wakeland and Hoarfrost 2005). During each validation task, three aspects of knowledge are involved, domain knowledge, inference knowledge and validation task knowledge. By analyzing the formation and function of each type of knowledge, knowledge model are constructed. These knowledge models, are typical to military simulation and independent of individual application. Based on these models, we can design prototype systems and knowledge base. As knowledge acquisition is proceed, any new knowledge and rules can be easily inserted with little modifications. In Min, Ma, and Yang (2007), we detail the knowledge elicitation and acquisition method with an example. In addition, we also discussed the complex characterization of military domain knowledge with some instances, the meaning of structural validity and behavioral validity.

From the introduction above, we can conclude the main challenges for knowledge-base validation of military simulation. First, domain knowledge elicitation and acquisition. This work can be very exhausting, and heavily dependent on application. As the simulation exercise continues, knowledge reuse and dealing with inconsistencies are also key problems confronted with knowledge acquisition. Second, construction of knowledge models, as the fact that military domain knowledge model can be comparatively complex, the inference models and validation task models should be independent of special application. Third, the design and implement of prototype system. Several issues involved within this problems, such as the design of knowledge base fit for the different kind of validation knowledge, the inference mechanism for the analysis and comparing task, etc. Another problem with this knowledge-based approach is that it is hard to design rational testing experiment. In military simulation, the number of entities and variables involved become comparatively great, and the conditions under which the model is running become more complex. This means it is hard to get enough testing data for each of the validation tasks in limited experiments. However, some literatures have discussed this problem in detail (Walton, Patton, and Parsons 2001).

\section{KNOWLEDGE MODEL FOR MILITARY SIMULATION VALIDITION}

The complex context of domain knowledge and their use in simulation validation have been detailed in above. However, what we need is not only a plain knowledge base contained all the knowledge involved in validation processes. We should refine these knowledge into elaborate structures, within each structure they have typical forms and be used for similar tasks. In knowledge engineering, the term knowledge model is often used to articulate this method (Giarratano and Riley 1998; Schreiber et al. 2003).

There are three knowledge categories in military simulation validation knowledge model, namely, domain knowledge, inference knowledge and task knowledge. Domain knowledge, as discussed above, detail the reference data and knowledge in specific validation application. The inference knowledge record the fundamental inference steps to use domain knowledge, they can be considered as the basic component of inference mechanism. And the task knowledge describe the inference goals of specific applications, and how to decompose the inference goals into several subtasks and inference steps.

\subsection{Domain Knowledge Model}

Domain knowledge describe the static information about of knowledge object in simulation validation. An important character of these information is the dependence among different information segments, which can be transformed into kinds of rules. It is these rules that make the dynamic inference and the generation of new information possible.

In military simulation validation, these information segments and their dependences can be very complex, however, they can be organized within some schemas according to their structures. As discussed above, there will usually be several validation tasks in each military simulation, each focus on some specific behaviors. For each of these task, a set of outputs should be considered. For example, in a interceptor weapon system validation, some important components and their relevant behaviors should be validated individually, such as the performance of radar system, the movement of the interceptor and the target, etc.. Each of these validation task, different sets of simulation outputs and behaviors will be collected and analyzed. Although the decision processes for which output sets to be collected will be quite different, they can all be considered as a mapping from validation domain to simulation output domain. In domain knowledge model, the rules with similar mapping relationships and structures are termed as rule type. The rule type for the above mapping can be described as:

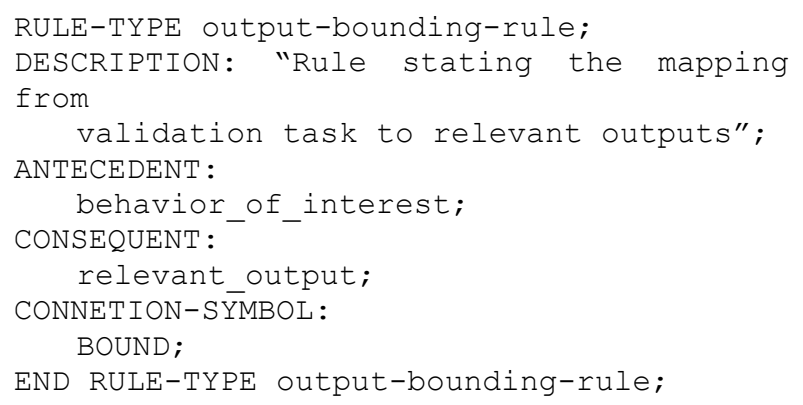




\section{Min, Ma, and Yang}

One of the main responsibilities of knowledge engineers is to analysis and extract all the rule types involved in simulation validation. Once the rule types are defined properly, the knowledge based can be designed. Each knowledge segment can be inserted in corresponding slot in the knowledge base in the form of instance. The following code shows part of the domain knowledge base used for the validation of interceptor weapon system simulation.

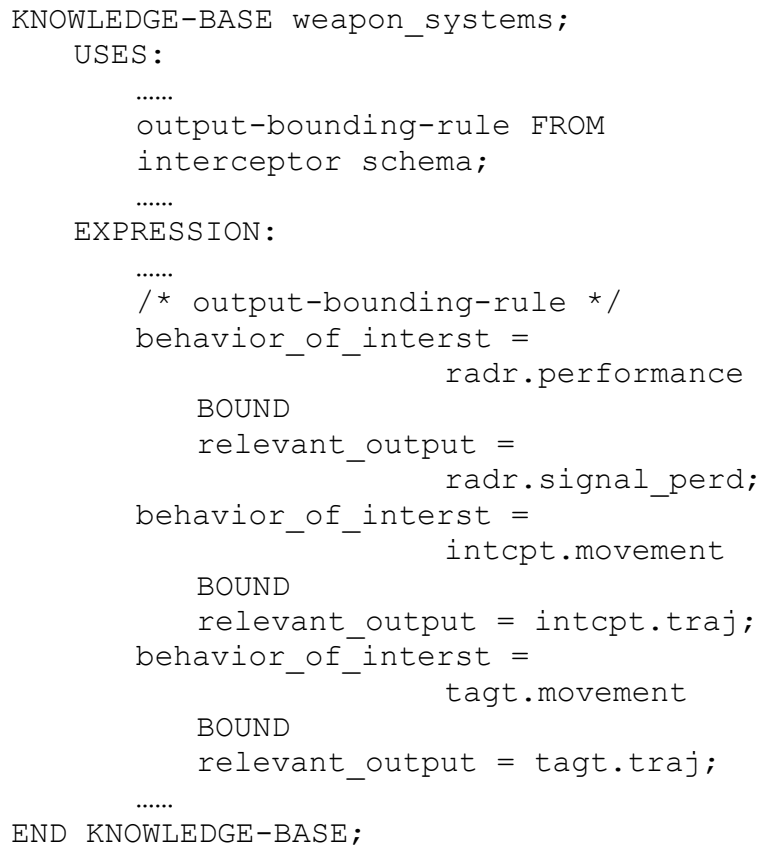

In a domain knowledge base, there are typically two kinds of slot, the USE slot is used to define rule types, and EXPRESSION for knowledge instances. Some more information about the rule types used in military simulation validation and the example of interceptor weapon system can be found in Min, Ma, and Yang (2007).

\subsection{Inference Knowledge Model}

The domain knowledge model discusses the description of static information/knowledge structure about application domain, while the inference knowledge model focuses on how to generate new information with these static knowledge. In knowledge model, an inference take the place as fundamental information process unit, and any complicated task can be accomplished by a sequence of inferences. On the other hand, these inference knowledge relate with the domain knowledge in some indirect way, which make the reuse of inference knowledge possible.

As it shows in a basic inference rule, there are typically two kinds of knowledge role in knowledge engineering, static role and dynamic role. The dynamic role terms the input and output of each inference, and different inferences employ different kinds of dynamic role instance. The static knowledge role is used to appoint the domain knowledge set that can be used in each inference, but not the domain knowledge itself. For example, the inference model describing all the output bonding rule instances can be coded as following:

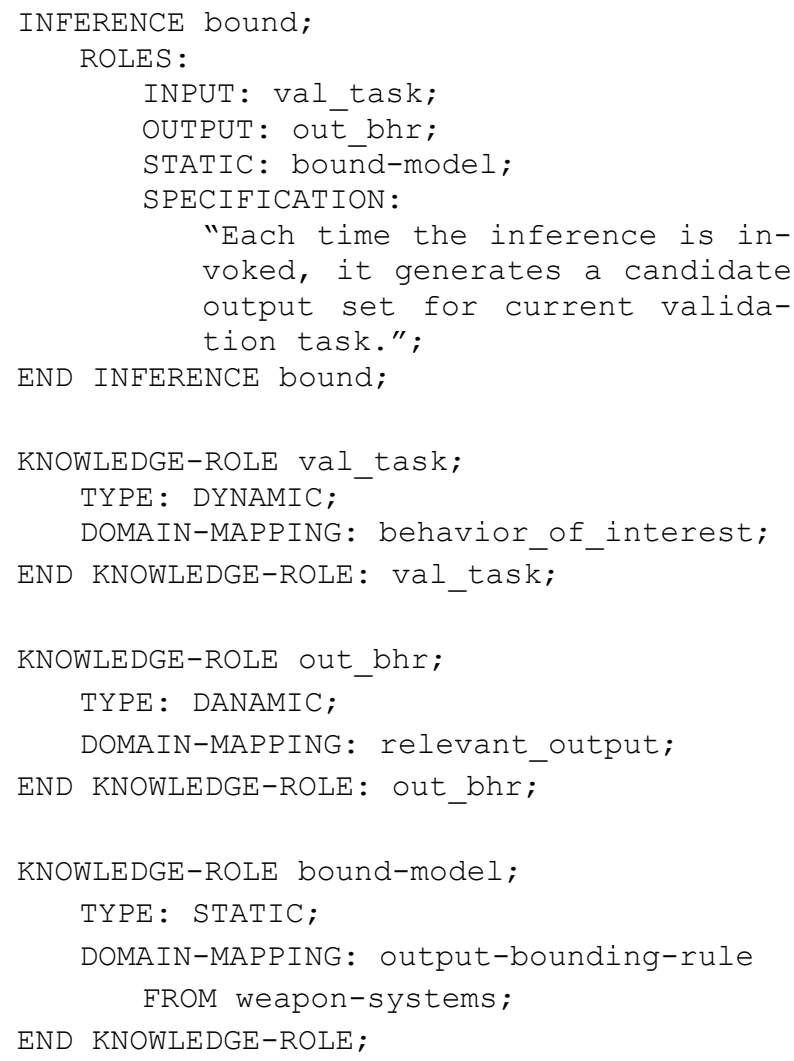

The terms dynamic and static roles make inference model decoupled with domain knowledge. It means that we can extract most of the inference models before all knowledge is available. It is this decoupling that makes prototype system is designed without consideration of the complex domain knowledge, and each kind of new knowledge can be insert to knowledge base conveniently.

\subsection{Task Knowledge Model}

In simulation validation, there are usually a sequence of works to be done before any validity decision is made, such as validation methods choosing, data analyzing, comparing, and validity deciding, etc.. The knowledge category task knowledge is used to describe the decomposing and scheduling of these works. High level and complicated tasks are decomposed into several low level and simple tasks, and the low level task can be also decomposed. This decomposing process should continue until no more task can be decomposed any more, each task can be decomposed into a sequence of inferences eventually by several task methods. 


\section{Min, Ma, and Yang}

As it shows in Figure 1, there are typically four top level subtasks for each validation task, i.e., selecting relevant knowledge segments, bounding corresponding behavior, extracting and analyzing simulation output and behavior of interest, evaluation and making validity decision. Some of these subtasks can also be decomposed. For example, there are often several different way to analyze simulation output, according to the type of domain knowledge to be used. We should also decompose the subtask extract/analyze in some proper way.

To realize the above decomposing process, some kind of task method can be employed. It decomposes the task with a sequence of functions, these functions may be some other subtasks or basic inference rules. In a task method, the control structure describe the scheduling of each subtask, and several subtasks take the place of parameters for each control structure. The following code realizes the task method for the decomposition in Figure 1.

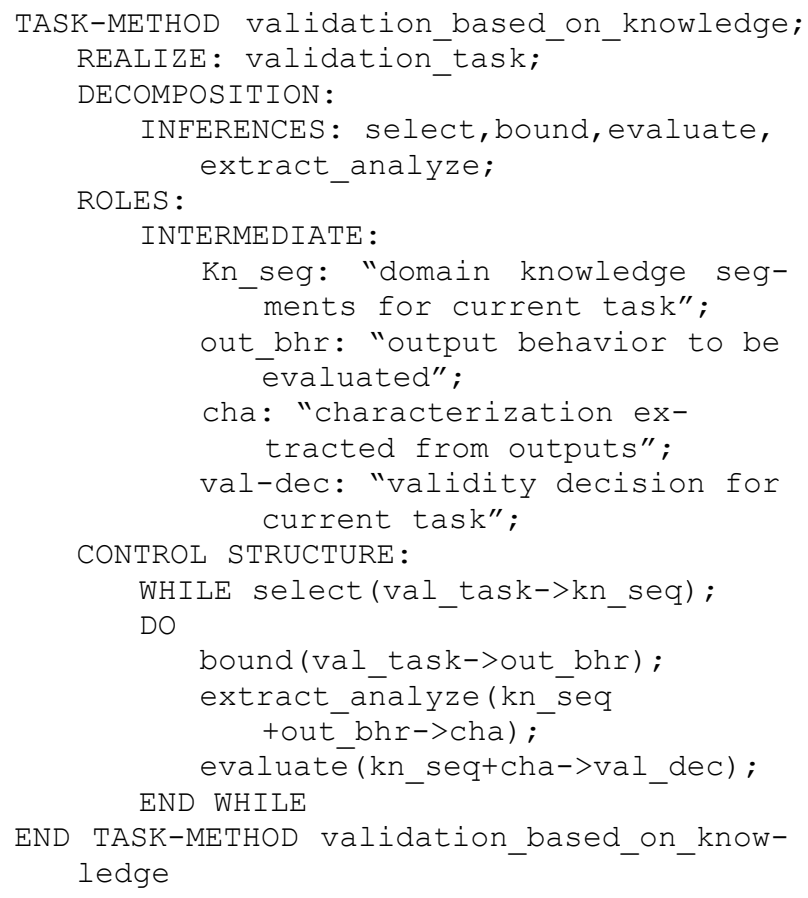

\section{IMPLEMENT OF KNOWLEDGE-BASED VALIDATION SYSTEM}

In this part, we will introduce the implement of knowledgebased system for military simulation validation. Although there are some requirements and constrains for this expert system, our discussion will only focus on the implement of the knowledge models.

The central issue involved in the design and implement process is the structure of the software. Schreiber (2003) gives a reference framework about general knowledgebased system. Figure 2 shows the components and their interactions for the implement of knowledge model in mili- tary simulation validation. There are nine main components in this framework, the dashed line shows the function transferring path, while the real line shows information access path.

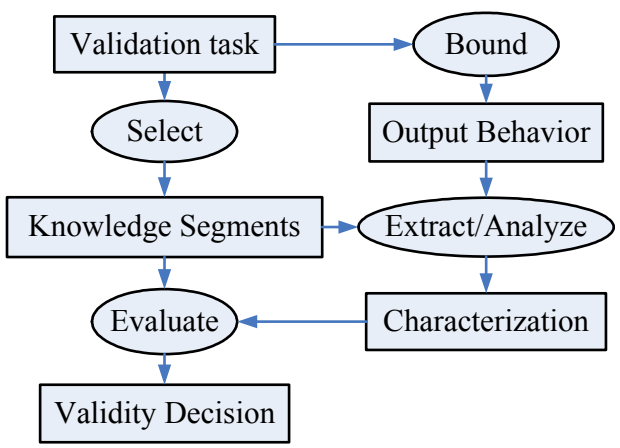

Figure 2: There are typically four top level subtasks for each validation task.

- Validation task: two operations should be defined for each validation task. first, initialization operation for each task, such as the illustration of the entities and behavior of interest in validation, validity requirements and some other related information. Second, the evoking of corresponding task methods.

- Task method: the main function of task method is to decompose each validation task into a sequence of fundamental inference. The task method is accomplished by hierarchical decomposing control structure. The management and access of these control structures is the main consideration of this component design. A inference list is employed for the scheduling of current validation task.

- Inference: as the validation task, inference objects are heavily dependent on the information contained in knowledge model. Each inference operation searches for dynamic and static role fit for current inference. If new solution is found, its related information are stored in some internal variables. And if no solution is found, the inference fails.

- Inference method: the inference component do not describe how the inference is accomplished. In this component, each inference is transformed into corresponding algorithm and variables. The knowledge and rules that match the description of corresponding dynamic and static roles are selected, and their parameters are insert to the inference algorithm.

- Dynamic role: as the input and output of each inference, there are two issues should be considered in the design of dynamic role component. First, the entities and interaction involved in military simulation can be comparatively complex, we should 
consider the data types and structures for the dynamic role design in detail. Second, the operations for each kind of data structures, such as access, update, and validation(to make sure that the data are complete and consistent).

- Static role: this component should support the access of each rule type and corresponding instances.

- Transform function: the transform function is designed to perform some particular task, it transforms information with component outside application model. There are mainly four kinds of task, namely, obtain, propose, receive and supply.

- Knowledge base: the information and rules about each entity and their interactions are stored in knowledge base structurally. As the data structures and relationships are more complex than that in general data base, the design of knowledge base can be much more difficult.

- Domain construction: the data and information from dynamic role, static role and knowledge base are integrated here.

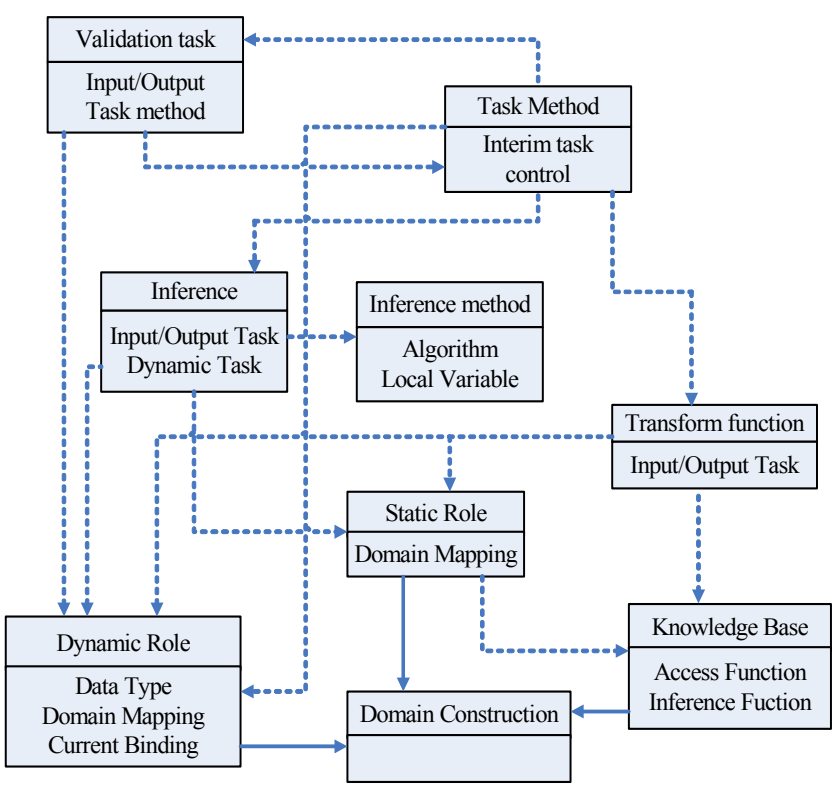

Figure 3: Framework of the knowledge-based system.

The main functions around the implement of knowledge model are introduced above. However, there are some other functions that should be considered. For example, the access and management of knowledge base, which is much more complex than general data base. That is because knowledge inserted should be complete, formal and consistent. Another important issues of the implement is around the user interface. As the validation process is advanced, the user may be interested in the task, inference and related instance employed currently. Especially, the system should display some key data, trajectories and other information as some important validity decision is made.
Figure 4 shows the main interface of validation user, it display the domain knowledge and inference structures used, and also specifies the relevant information generation process during each validation task.

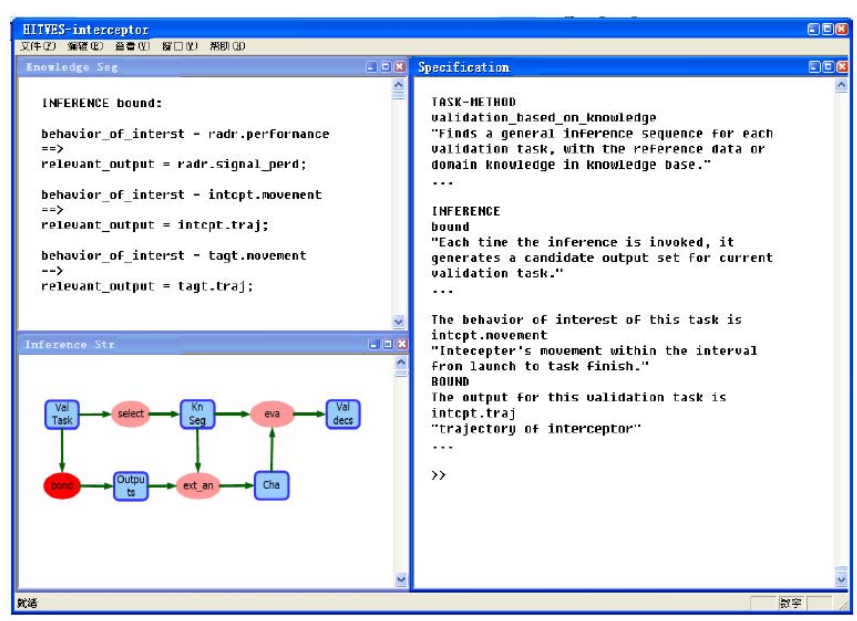

Figure 4: User interface of validation system.

We have discussed the implement of our knowledgebase validation system. However, this system should be enriched with enough knowledge before application. These enriching may involved some fatal modification, especially the domain knowledge model and its implement.

\section{CONCLUSION}

In this paper, the implement of knowledge-based system for the validation of military system is detailed. It focus on the construction of knowledge models and the implement of these models.

There are several key issues for future research, such as the elicitation and acquisition of miliatry domain knowledge, the maintance and management of knowledge base, enriching this system for practical application. In addtion, this design schema is general to most of complex system simulation validation, it can be adapted for some more application areas.

\section{ACKNOWLEDGMENTS}

This paper is supported by Natural Science Foundation of China (60434010) and the Foundation of the Outstanding Youth of Heilongiiang Province (JC200606).

\section{REFERENCES}

Balci, O. 1998. Verification, validation, and testing. In The Hand book of Simulation, 335-393. New York, NY: John Wiley \& Sons.

Balci, O. 1989. How to access the acceptability and credibility of simulation results. In Proceedings of the 1989 
Winter Simulation Conference, 62-71. Washington, DC: IEEE.

Barlas, Y. 1996. Formal aspects of model validity and validation in system dynamics. System Dynamic Review. 183-210.

Barlas, Y. and K. Kanar. 1999. A dynamic pattern-oriented test for model validation. in Proceedings of $4^{\text {th }}$ System Science European Congress. 269-286.

Birta, L. G. and F. N. Ozmizrak. 1996. A knowledge-based approach for the validation of simulation models: the foundation. ACM Transactions on Modeling and Computer Simulation, 6(1): 76-98.

Courtois, P. J. 1985. On time and space decomposition of complex structures. Communication of the ACM. June: 590-603.

Defense Modeling and Simulation Office (DMSO). 2001. Department of defense verification, validation, and accreditation recommended practices guide. Department of Defense, Alexandria, VA.

Fang, K., M. Yang, and Z. Wang. 2005. The HITVICE VV\&A environment. in Proceeding of Winter Simulation Conference, 1220-1227.

Giarratano, J. and G. Riley. 1998. Expert Systems Principles and Programming. PWS Publishing Company. 217-230.

Hofmann, M. A. 2004. Criteria for decomposing systems into components in modeling and simulation: lessons learned with military simulations. SIMULATION: the Society for Modeling and Simulation International. JulAug, 357-365.

Hopkinson, W. C. and J. A. Sepulveda. 1995. Real time validation of man-in-the-loop simulations. In Proceedings of the 1995 Winter Simulation Conference. 12501256.

Kleijnen. J. P. C. 1999. Validation of models: statistical techniques and data availability, Proceeding of Winter Simulation Conference, 647-654.

Law, A. M. and W. D. Kelton. 2000. Simulation modeling \& analysis. 3rd ed. New York: McGraw-Hill, Inc.

Min, F., P. Ma, and M. Yang. 2007. Domain knowledge elicitation and acquisition for military simulation. Submitted to Proceedings of Winter Simulation Conference.

Findler, N. V. and N. M. Mazur. 1990 A system for automatic model verification and validation. Transactions of the Society for Computer Simulation International. 6(3): 153-172.

Sargent., R. G. 2004. Verification and validation of simulation model. in Proceeding of Winter Simulation Conference, 17-28.

Schreiber, G., H. Akkermans, A. Anjewierden, R. deHoog, N. Shadbolt, W. VandeVelde, and B. Wielinga. 2003. Knowledge Engineering and Management: the CommonKADS Methodology. 85-120. MIT Press.
Song, H., M. Yang, and P. Ma. 2005. The application of evaluation method based on HMM for Result validation of complex simulation system. In Proceeding of the 1995 Winter Simulation Conference, 1228-1233.

Wakeland, W. and M. Hoarfrost. 2005. The case for thoroughly testing complex system dynamics.

Walton, G. H., R. M. Patton, and D. J. Parsons. 2001. Usage testing of military simulation systems. In Proceeding of the 2001 Winter Simulation Conference.

Zeigler, B. P. 1987. Hierarchical, modular discrete-event modeling in an object-oriented environment. SIMULATION. 249-230.

Zhang, B., M. Yang, and B. Li. 2002. Integration of testing and evaluation for complex simulation system, Computer Integrated Manufacturing Systems 8(3).

\section{AUTHOR BIOGRAPHIES}

FEIYAN MIN is now a Ph.D. candidate majoring in modeling and simulation of complex system. His research interests include modeling, validation and calibration of complex systems . His email address is <minfeiyandyahoo.com.cns.

PING MA is an Associate Professor at Harbin Institute of Technology. She received her education at Harbin Institute of Technology. Her research interests include system simulation, VV\&A. Her email address is $<$ pingma@hit. edu. cn>.

MING YANG is a Professor at Harbin Institute of Technology. He received his education at Harbin Institute of Technology. His research interests include system simulation, system control and VV\&A. His email address is $<$ myangahit. edu. cn> . 